\title{
Role of nonreinforcement in the fixed-interval performance of pigeons
}

\author{
MAURICIO R. PAPINI and PETER R. HOLLINGSWORTH \\ Texas Christian University, Fort Worth, Texas
}

\begin{abstract}
Fixed-interval (FI) schedules have been used extensively to study timing abilities. In FI schedules, animals typically show higher response rates immediately after nonreinforced $(\mathrm{N})$ cycles rather than reinforced $(R)$ cycles (the reinforcement-omission effect), and they exhibit the highest rate approximately at the time when the reinforcer is scheduled to occur (peak performance). The present experiments were designed to determine the extent to which factors other than timing contribute significantly to these two learning phenomena. Pigeons were trained in an FI 16-sec schedule in which half the cycles were $R$ and half were $N$. When successive cycles were separated by a 2-sec interval, responding early in the FI interval was higher after an $\mathrm{N}$ cycle than after an $\mathrm{R}$ cycle. This reinforcementomission effect was eliminated when the interval between cycles was increased to $12 \mathrm{sec}$, because of an increase in performance after $R$ cycles. In addition, timing of the 16-sec interval was assessed by interpolating 32-sec test cycles (all N cycles) at two rates-either 1 test cycle every other session, or 25 test cycles per session. Peak performance, presumably indexing the animal's ability to time the 16-sec interval, emerged only with 25 test cycles per day, but not with 1 test cycle every other day, despite extensive training with the target, 16-sec-long interval. These results suggest that transient demotivation and time-based discrimination contribute significantly to the reinforcement-omission effect and peak performance, respectively.
\end{abstract}

Arranging a regular distribution of reinforcers in time, as in a fixed-interval (FI) schedule of reinforcement, is a procedure that has been used extensively to study the timing abilities of organisms (Lejeune \& Wearden, 1991). It has long been recognized that unexpected nonreinforced $(\mathrm{N})$ cycles are followed by higher response rates than reinforced $(\mathrm{R})$ cycles are (Staddon \& Innis, 1966). This phenomenon, referred to as the reinforcementomission effect, has been linked to a variety of mechanisms, including the role of reinforcement in resetting the internal clock used to estimate the duration of the interval (Mellon, Leak, Fairhurst, \& Gibbon, 1995). However, as Mellon et al. noted, when the reinforcement and other associated cues are omitted, animals face what is essentially a longer FI cycle. This situation approaches the conditions of the peak procedure, in which an unusually long test cycle is interpolated with shorter training cycles to assess the animal's ability to estimate the duration of the short interval. Animals exhibit peak performance - that is, a maximum response rate in the long test cycle at approximately the time within the interval when reinforcement is usually scheduled to occur (S. Roberts, 1981). In the present experiments, we examined the contribution of factors other than timing to these learning phenomena.

The research reported in this paper was partially supported by TCU/RF Grant 5-23832 to M.R.P. Requests for reprints may be sent to $M$. R. Papini, Department of Psychology, Texas Christian University, TCU Box 298920, Fort Worth, TX 76129 (e-mail: papini@gamma.is.tcu.edu).

\section{EXPERIMENT 1 Reinforcement-Omission Effect}

Several hypotheses have been proposed to explain the reinforcement-omission effect. Staddon and Innis (1969; Staddon, 1970) suggested that response rate increases because the omission removes the inhibitory influence of reinforcement on performance established as a result of extensive exposure to the FI schedule in which the reinforcer is consistently followed by a period of nonreinforcement. Supporting evidence for this disinhibition view comes from a manipulation of the degree of similarity in stimulus conditions between $\mathrm{R}$ and $\mathrm{N}$ cycles. Kello (1972) trained pigeons in an FI 2-min situation and reinforced them with access to grain for $3 \mathrm{sec}$. Together with grain presentation, the houselight was turned off (blackout) and a magazine light was turned on. Kello argued that pigeons responded more after $\mathrm{N}$ cycles ending in blackout alone than after $\mathrm{N}$ cycles ending in blackout plus magazine light, because the magazine light had acquired inhibitory control on keypecking during $\mathrm{R}$ cycles.

Both Staddon (1970) and Kello (1972) were testing this disinhibition hypothesis against Amsel's frustration theory (see Amsel, 1992). According to frustration theory, the unexpected omission of a reward induces an emotional reaction, termed primary frustration, that facilitates ongoing behaviors (Papini \& Dudley, 1997). Application of frustration theory to Kello's study predicted that the greater the similarity between $\mathrm{N}$ and $\mathrm{R}$ situations, the greater the surprising effect of reward omission, the level of primary frustration, and the facilitation of performance. 
Frustration theory wrongly predicted a higher response rate after blackout plus magazine light than after blackout alone, because the first situation was more similar to the context in which reward was presented during $\mathrm{R}$ cycles.

A third possibility is that reinforcers play a direct role in timing. For example, Mellon et al. (1995) argued that increased responding after unexpected reward omissions in FI schedules may reflect lack of precision in estimating the interval to the next reinforcement. According to this view, an accurate temporal discrimination of the $\mathrm{FI}$ value depends on the internal clock's being reset by reinforcement at the end of each cycle. When only the cues paired with reinforcement are presented, but not the reinforcer itself, the resetting process is only partially accomplished, leading to imprecision in timing.

In addition to disinhibition, primary frustration, and timing, it is possible that performance early in the FI cycle is depressed after an $R$ cycle by a transient state of demotivation, rather than increased after an $\mathrm{N}$ cycle by any of the other mechanisms. Although demotivation cannot have been a factor in Kello's (1972) experiment, in which the critical comparison was made between two types of $\mathrm{N}$ cycles, it remains a viable account when comparisons involve $\mathrm{N}$ versus $\mathrm{R}$ cycles.

In the present experiment, these four hypotheses of the reinforcement-omission effect were tested by manipulating the interval between successive FI cycles across phases. The introduction of an interval separating successive FI cycles transforms this procedure into a discrete-trial situation, and thus the interval will be referred to as the intertrial interval (ITI). During the ITI, the conditions of stimulation remained constant except that the keylight was turned off. A comparison of asymptotic performance with either a $2-\sec$ or a $12-\sec$ ITI provided the primary data.

In the case of the disinhibition hypothesis, since the reinforcer predicts a period of nonreinforcement whether the ITI is $2 \mathrm{sec}$ long or $12 \mathrm{sec}$ long, performance was expected to be higher after $N$ than after $R$ cycles at both ITI values. If anything, an ITI of $12 \mathrm{sec}$ should be accompanied by stronger inhibition accrued to the reinforcer, because it predicts a longer nonreinforced period than in the 2 -sec case. Thus the disinhibition hypothesis would be consistent with an increase in the reinforcementomission effect in the 12 -sec condition in comparison with the 2 -sec condition. In the case of the primary frustration hypothesis, since it is assumed to be a transient emotional state (Dudley \& Papini, 1995), the difference in performance after $\mathrm{N}$ versus $\mathrm{R}$ cycles should diminish or completely disappear after a shift from an ITI of $2 \mathrm{sec}$ to one of $12 \mathrm{sec}$. However, the primary frustration hypothesis predicts that this effect should be caused by a decrement in performance after $\mathrm{N}$ cycles, rather than an increment in performance after R cycles; an increase in the ITI would allow for a substantial decay of primary frustration by the onset of the following FI cycle. According to Mellon et al.'s (1995) timing hypothesis, the internal clock is reset by reinforcement, a plausible assumption when the procedure involves no stimulus change from one cycle to the next, as was the case in their experiment. The assumption is less tenable when an explicit ITI period is interpolated between successive cycles, as was done in the present procedure. In this case, it seems likely that the hypothetical internal clock is reset by keylight onset, rather than by reinforcement, and thus the theory would predict no reinforcement-omission effect under either 2- or 12-sec ITI training. A low response rate in the initial portion of the FI cycle would suggest that keypecking was under the control of keylight onset. Finally, the demotivation hypothesis predicts that an increase in the ITI from 2 to $12 \mathrm{sec}$ should lead to the reduction or disappearance of the reinforcement-omission effect. However, unlike in the primary frustration account, this ITI effect should be the result of an increase in the response rate after $\mathrm{R}$ cycles following a shift from an ITI of $2 \mathrm{sec}$ to one of $12 \mathrm{sec}$. The increased ITI value should allow for the dissipation of transient satiety after an $\mathrm{R}$ cycle so that performance would recover to a level similar to that observed after $\mathrm{N}$ cycles.

\section{Method}

Subjects. The subjects were 15 pigeons, all sexually mature. They were obtained from Rurhardt Pet and Feed, Fort Worth, and had been previously trained in various keypecking situations with pellets serving as the reward (45-mg Noyes pellets, pigeon formula). Water was continuously available in the individual cages. The pigeons were kept at approximately $75 \%$ of their free-feeding weights. The colony room was continuously illuminated.

Apparatus. The pigeons received training in three identical Skinner boxes. Each box was enciosed in a sound-attenuating chamber equipped with a fan that provided ventilation and masking background noise. Each conditioning box measured $32.2 \times 29.9 \times 32.2 \mathrm{~cm}$ (width $\times$ length $\times$ height). The front wall, back wall, and ceiling were made of clear Plexiglas, whereas the two lateral walls were made of aluminum. One of the lateral walls contained the feeder cup $(3 \mathrm{~cm}$ above the floor, in the center of the wall), a lamp (General Electric 1820) providing diffuse illumination from the upper left corner, and the response key $(1.8 \mathrm{~cm}$ in diameter, $18.5 \mathrm{~cm}$ above the floor, in the center of the wall). The feeder cup was made of opaque Plexiglas and was $4.5 \times 5.5 \times 4 \mathrm{~cm}$. Noyes precision pellets (pigeon formula, $45 \mathrm{mg}$ ) were automatically delivered into this cup. A white light projected on the response key served as the discriminative stimulus during training. All stimulus events were controlled by a computer located in an adjacent room, which also recorded the keypecking responses.

Procedure. The pigeons were extensively pretrained in the keypecking situation. During the initial 10 sessions, the subjects received training on a discrete trial situation. In each trial, the white light was turned on for a maximum of $6 \mathrm{sec}$; a food pellet was delivered either when the pigeon pecked at the key, or at the end of the trial if no keypecks had been detected by the computer. There were 50 trials in each session, separated by a variable ITI (mean, $30 \mathrm{sec}$; range, 15-45). All subjects were subsequently exposed to a series of increasing FI requirements. From Day 11 to Day 14 , they were exposed to an FI 5-sec schedule; on Days 15-16, to FI 7 sec; on Days 17-19, to FI $10 \mathrm{sec}$; on Days 20-21, to FI $13 \mathrm{sec}$; and on Days 22-23, to FI $16 \mathrm{sec}$. In all of these sessions, there were $50 \mathrm{FI}$ cycles separated by an ITI of $2 \mathrm{sec}$. Each cycle started when the white keylight was turned on; responses during the cycle had no scheduled consequence, but the first keypeck occurring after the cycle had reached the required duration caused the delivery of a single food pellet and turned off the white keylight. The keylight remained off during the ITI. The houselight was on during the entire session. Each session started when the houselight was turned on; a 2 sec interval similar to the ITI followed. Sessions ended with a 2-sec interval following the last cycle after which the houselight was turned off 
Phase 1 of the main part of this experiment began on Day 24 and ended on Day 77. Before the start of Phase 1, the subjects were assigned to one of the two groups on the basis of their mean response rates over the last two sessions of pretraining with the FI 16-sec schedule. Pairs of pigeons matched in their mean response rate were formed, and individual birds were randomly assigned to one of the two groups. The mean responses per minute were 18.3 (range, 8.5-26.7) for the pigeons assigned to Group C, and 19.5 (range, 8.4-32.5) for the pigeons assigned to Group P. Because of unequal sample size, 1 pigeon (averaging 21.3 responses per minute and assigned to Group P) did not have a matched bird. Group C (i.e., continuous, $n=7$ ) was scheduled to receive reinforcement at the end of each FI cycle; this is the typical training procedure in experiments involving FI schedules and was included to provide an independent baseline. Group P (i.e., partial, $n=8$ ) was scheduled to receive reinforcement on a random $50 \%$ of the cycles. This was the critical group as far as the predictions outlined in the introduction are concerned. There were $25 \mathrm{R}$ and $25 \mathrm{~N}$ cycles per session. On a nonreinforced cycle, the first response emitted after the end of the interval turned off the keylight and initiated the ITI; no pellet was delivered. Starting on Day 55, and on every other day during the rest of Phase 1, sessions also included a test cycle, randomly placed between cycles 40 and 50 within each session. The test cycles lasted $32 \mathrm{sec}$, always ended in nonreinforcement, and they were relevant to the results reported in Experiment 2.

Phases 2 and 3 were each 20 sessions long. Phase 2 was scheduled on Days 78-97. The scheduled events were the same as during Phase 1, except that the ITI was increased to $12 \mathrm{sec}$. Phase 3 was run on Days 98-117. Scheduled events in Phase 3 were exactly the same as in Phase 1. Phases 1-3 therefore constituted an A-B-A design, with ITI ( 2 vs. 12 sec) as the independent variable.

Across all phases of the experiment, each session started with a warmup $\mathrm{R}$ cycle which was not counted as one of the 50 cycles per session. Responses generated during this warm-up cycle were not included in the analyses. The dependent measure was the within-cycle response rate, computed on 1-sec bins, averaged over the last 10 sessions of each phase for each pigeon.

\section{Results}

Figure 1 shows the mean within-cycle performance during the final 10 sessions of Phases $1-3$. Table 1 presents the same results but averaged for the entire cycle; although the statistical analyses were performed on the data plotted in Figure 1, the summary provided by Table 1 shows some of the phase differences more clearly. For Group P, FI cycles were segregated according to the outcome of the prior cycle, either after R or after N. For Group $C$, the curves represent the average session performance. Since the number of cycles per session was equated across groups, the functions for Group $\mathrm{P}$ were computed on the basis of lower number of cycles relative to Group C's functions. As Figure 1 indicates (see also Table 1), a small but consistent increase in response rate was obtained in cycles following $\mathrm{N}$, relative to those following R, in Group P during Phases 1 and 3. The difference disappeared in Phase 2, when the ITI was increased from 2 to $12 \mathrm{sec}$. Less obvious in Figure 1 is the rise in performance level during Phase 2, compared with Phases 1 and 3 (see overall cycle averages in Table 1). The main analysis of variance involved three factors, all repeated measures: cycle (after $\mathrm{R}$, after $\mathrm{N}$ ), phase, and 1sec bin. There were significant simple main effects of cycle $[F(1,665)=8.77, p<.01]$, phase $[F(2,665)=$ $17.16, p<.001]$, and $1-\sec$ bin $[F(15,665)=166.22$, $p<.001]$. The disruption of the reinforcement-omission effect during Phase 2 was confirmed by a significant cycle $\times$ phase interaction $[F(2,665)=4.34, p<.025]$. All other interactions were nonsignificant. Thus, the manipulation of increasing the ITI in Phase 2 was successful in disrupting the reinforcement-omission effect.

The next question was whether the disruption was caused by a performance decrease after $\mathrm{N}$ cycles (primary frustration hypothesis), or by a performance increase after R cycles (demotivation hypothesis). Separate phase $\times 1$-sec bin analyses of Group P's data were performed for the cycles following $\mathrm{N}$ and following $\mathrm{R}$, across phases. In both analyses there was a highly significant $1-\mathrm{sec}$ bin effect $[F \mathrm{~s}(15,329)>73.08, p \mathrm{~s}<$

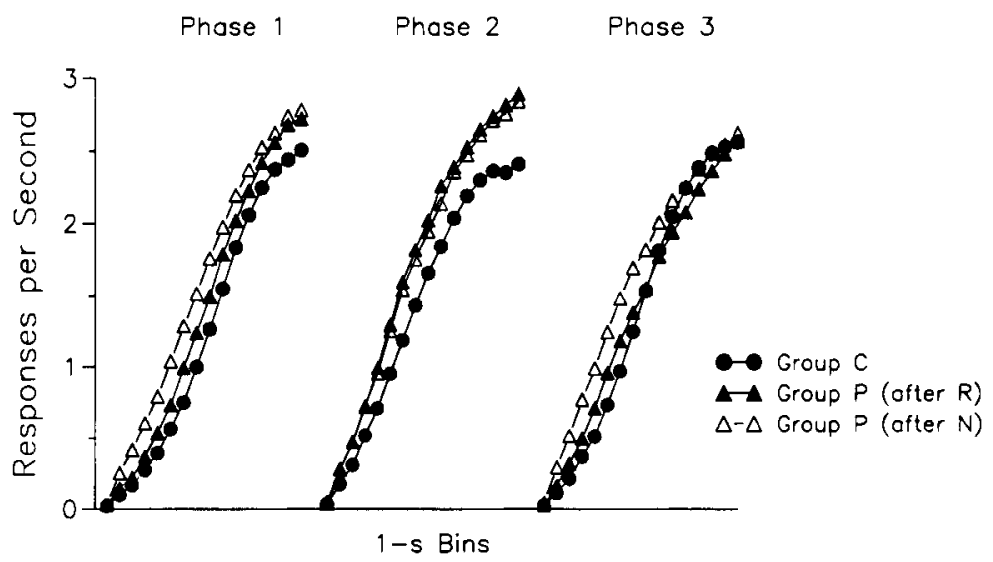

Figure 1. FI performance of both groups during Phases 1-3. ITI was 2 sec long in Phases 1 and 3, and 12 sec long in Phase 2. Group $C$ received 50 FI 16-sec cycles per session, all ending in reinforcement. Group $P$ received 50 FI 16-sec cycles per session, 25 ending in reinforcement and 25 ending in nonreinforcement. The performance of Group $P$ is segregated according to whether the previous cycle ended in reinforcement (after $\mathbf{R}$ ) or in nonreinforcement (after $\mathbf{N}$ ). Responses per second were accumulated every 1-sec bin within the FI 16-sec interval. Each data point is the mean of the last 10 sessions of each phase for that particular bin. 
Table 1

Mean Responses (With Standard Deviations) per Second and per FI Cycle During the Last 10 Sessions of Each Phase

\begin{tabular}{lccccccc}
\hline & \multicolumn{2}{c}{ Phase 1 } & & \multicolumn{2}{c}{ Phase 2 } & & \multicolumn{2}{c}{ Phase 3 } \\
\cline { 2 - 3 } \multicolumn{1}{c}{ Condition } & $M$ & $S D$ & & $M$ & $S D$ & $M$ & $S D$ \\
\hline Group P, after R & 1.39 & 1.1 & 1.72 & 1.2 & 1.39 & 1.3 \\
Group P, after N & 1.56 & 1.1 & 1.68 & 1.2 & 1.58 & 1.2 \\
Group C & 1.22 & 1.0 & 1.41 & 0.9 & 1.36 & 1.0 \\
\hline
\end{tabular}

$.001]$. The phase effect, however, was significant for the cycles following $\mathrm{R}[F(2,329)=16.57, p<.001]$, but not for the cycles following $\mathrm{N}[F(2,329)=2.64]$. In both cases, the overall response rate means for the entire cycle exhibited an increase in Phase 2, compared with Phases 1 and 3 , as can be seen in Table 1 . These results provide support for the demotivation hypothesis, which predicted a disruption of the reinforcement-omission effect as a result of an increase in response rate after $R$, when pigeons were shifted from an ITI of $2 \mathrm{sec}$ to one of $12 \mathrm{sec}$. Primary frustration predicted the disruption to arise out of a fall in response rate in the cycles following $\mathrm{N}$ during Phase 2. However, the performance after $\mathrm{N}$ cycles actually increased during Phase 2, relative to Phases 1 and 3 , suggesting that primary frustration did not contribute significantly to the present results.

The performance of Group $\mathrm{C}$ is also instructive for two reasons. First, although Figure 1 suggests that Group C's performance was below that of Group P during Phase 2, a comparison of Group $C$ and Group $P$ after $N$ performance across phases and 1 -sec bins indicated only a significant change within the cycle $[F(15,585)=308.91$, $p<.001]$. All other simple effects and interactions were nonsignificant. Second, the performance of these pigeons also increased during Phase 2, relative to Phases 1 and 3 (see Table 1 ), and it did so significantly $[F(2,282)$ $=10.49, p<.001]$. This increase of performance in Phase 2 when the ITI was lengthened to $12 \mathrm{sec}$ is also consistent with a demotivation account of the reinforcementomission effect.

One aspect of the results described in Figure 1 that differs from analogous published results (e.g., Mellon et al., 1995 ) is the low response rate early in the FI interval after an $\mathrm{N}$ cycle, surely related to the use of an ITI (i.e., an interval during which the stimulus conditions change relative to those enforced during the FI cycles). This differs from a strictly free-operant procedure in which the transition from one cycle to another is not obvious in $\mathrm{N}$ cycles because there is no stimulus change (cf. Mellon et al., 1995). Operant behavior was under tight discriminative control by the white keylight in the present training conditions. This makes it reasonable to assume that timing (i.e., starting the internal clock) was likely under the control of the keylight, rather than of the outcome of the previous cycle. As we argued above, the timing hypothesis predicts no reinforcement-omission effect under these conditions because it would be the onset of the key- light stimulus (and not the reinforcer) that resets the internal clock. The present results are therefore inconsistent with the disinhibition, primary frustration, and timing hypotheses, although for different reasons, and they provide support for a demotivational account of the reinforcementomission effect.

\section{EXPERIMENT 2 Peak Performance}

When reinforcers are omitted in the course of FI training, the subject faces what in fact is a longer time interval. As we noted earlier, such mixture of FI reinforced trials with longer test cycles is one procedure commonly used to assess the timing abilities of animals- - the socalled peak procedure (S. Roberts, 1981). In a typical timing situation, animals are exposed to two types of cycles. Training cycles involve the duration to be discriminated and typically end in reinforcement, whereas test cycles are longer than training cycles and typically end in nonreinforcement. The proportion of training-to-test cycles varies across experiments, with typical values ranging from 80:20 (S. Roberts, 1981) to 75:25 (W. A. Roberts, Cheng, \& Cohen, 1989) and 50:50 (Mellon et al., 1995). Test cycles provide evidence of timing in the form of a distribution of responses peaking near the FI value used in training cycles and referred to as peak performance. The implication is that animals have learned to discriminate the duration of the interval, and when the reinforcer fails to occur at the predicted time, response rate simply decreases gradually because of temporal generalization decrement. It is possible, however, that factors other than pure timing contribute to the decrease in response rate observed after the training FI value.

Given the consistent nonreinforcement of test cycles, potential factors involve a time-based discrimination, based either on reduced generalized excitation or on inhibitory conditioning, and anticipatory frustration. According to a reduced generalized excitation hypothesis, the response decrement observed after the FI value depends on the extinction of excitation generalized from stimuli related to the initial part of the cycle (the $S+$ in this case) to stimuli related to the final part of the cycle (the $\mathrm{S}-$ ). Such extinction would be a consequence of the repeated nonreinforcement of test cycles. Alternatively, the reduction of performance later in the FI cycle could result from inhibitory conditioning developing to $\mathrm{S}-$. 
Cues related to the final part of the cycle would actually suppress performance as a result of a summation of generalized excitation from $\mathrm{S}+$ and inhibition from $\mathrm{S}-$. A third account of peak performance, based on anticipatory frustration, suggests that decreased responding later in the FI cycle reflects anticipation of a hypothetical frustrative response produced by nonreinforcement in relatively long FI intervals.

By contrast to simple temporal generalization, these three alternative hypotheses of peak performance depend on the subject's receiving substantial exposure to $\mathrm{N}$ cycles within the session. These three hypotheses predict a greater performance decrement after the FI value as the number of training cycles approximates the number of test cycles, although perhaps the reduced generalized excitation one could be considered the most parsimonious of the three because it requires no assumptions regarding inhibitory learning or the anticipation of an emotional response of frustration. The present experiment was not designed to distinguish between these three possibilities, but only to provide evidence as to whether or not peak performance is the result of mere time generalization decrement. A time generalization account predicts that the accuracy of timing, and hence the amount of decrement after the FI value, should depend more on the number of training cycles than on the number of test cycles. These predictions were assessed by shifting, across phases, the numbers of both training and test trials per session.

\section{Method}

Subjects and Apparatus. The subjects and apparatus from Experiment 1 were again used in Experiment 2.

Procedure. Two more phases were added to the three described earlier. each 20 sessions long. Phase 4 lasted from Day 118 through 137. During this phase, the proportion of test cycles was increased from 1 cycle every other day to 25 cycles per session. These test cycles continued to be $32 \mathrm{sec}$ long, $\mathrm{N}$ cycles. The 25 training cycles were all $\mathrm{R}$ cycles for Groups $\mathrm{C}$ and P. During Phase 5, Days 138-157, the conditions of training were the same as in Phase 3. Phases 3-5 constituted, therefore, an A-B-A design, with proportion of training-to-test cycles per session as the independent variable, and with number of total cycles per session constant. All other aspects were as described for Experiment 1 .

\section{Results}

Although the critical phase for this experiment was Phase 4, when the proportion of test cycles was increased, test cycle data had been collected in each of the five phases of training. In all phases, the mean response rate was higher for Group P than for Group C, but analyses for each phase indicated nonsignificant group effects $[F \mathrm{~s}(1,13)<3.68, p>.05]$. The largest $F$ value occurred in Phase 2 when the ITI was $12 \mathrm{sec}$, a manipulation that is irrelevant with regard to peak performance. Therefore, the data from both groups were pooled for the purpose of statistical analyses. Figure 2 shows the test performance of all the pigeons for each phase of the experiment. During Phases 1, 2, 3, and 5 there was a single test cycle every other day. Each of these functions represents the mean performance of 5 test cycles, distributed over the last 10 days of each phase. The function for Phase 4 represents the average performance of all pigeons also during the last 10 days of training in this phase. There were 25 test cycles per day, however, so each pigeon contributed 250 cycles to this function.

The most important and obvious result reported in Figure 2 is the fall in response rate during the second half of the 32-sec test cycle obtained in Phase 4. This approximates a typical peak performance, although response decrement in the present experiment was not as pronounced as in some published experiments (e.g., Roberts, 1981). The function peaked, nonetheless, at the 16 th second. Individually, 4 of the 15 animals actually

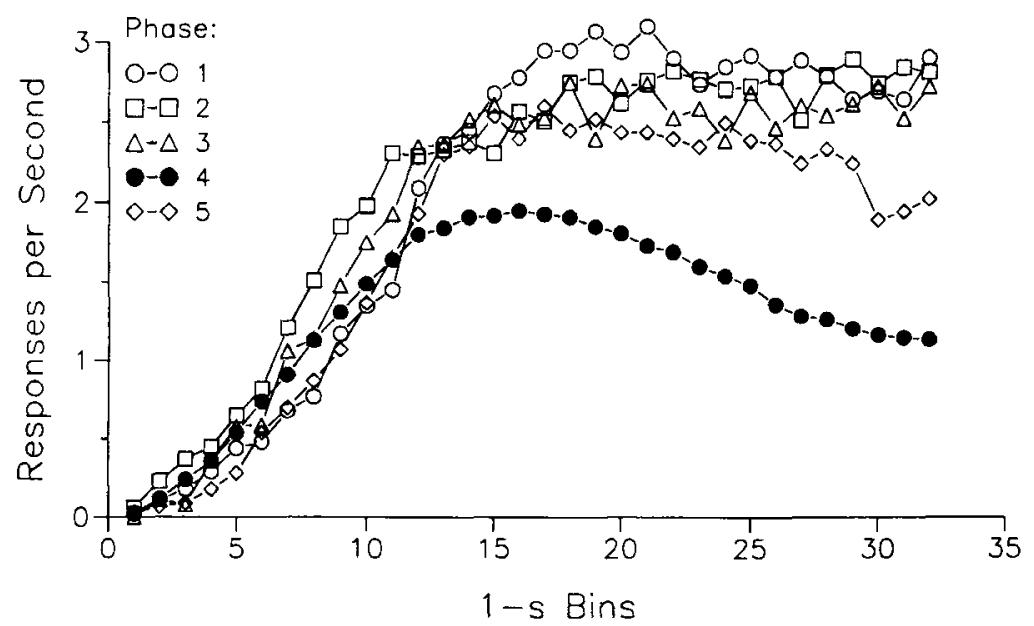

Figure 2. Average response rate during the 32-sec-long test cycles in each of the five phases of training. The performance of all the subjects in the experiment was pooled to produce these functions. The functions for Phases 1-3 and 5 were obtained by averaging 5 test cycles distributed over the last 10 sessions of each phase for each subject. The functions for Phase 4 represent the mean of 250 cycles, 25 cycles in each of the last 10 sessions of the phase, for each subject. 
peaked at the 16 th second, 4 more at the 17 th second, 2 at the 18th second, and 1 pigeon peaked at each of the following intervals within the $32-\mathrm{sec}$ test cycle: $12 \mathrm{th}$, 13th, 14th, 15th, and 20th. Also critical for the present purpose is the absence of any indication of peak performance in any of the other phases of the experiment, when there was only one test cycle every other session. All of the functions show an increase in performance that levels off after the 16th second; only the function for Phase 5 shows a tendency for performance to decrease toward the end of the cycle. An analysis of these data in terms of a phase $\times 1$-sec bin model, with repeated measures for both factors, yielded highly significant effects of phase $[F(4,2226)=135.11, p<.001]$ and $1-\mathrm{sec}$ bin $[F(31,2226)=150.43, p<.001]$, and a significant interaction $[F(124,2226)=3.55, p<.001]$. Peak performance is therefore more likely to emerge when there is a greater proportion of test cycles, even if there is a concomitant reduction in the proportion of training cycles, than simply as a function of the amount of training with the time interval to be estimated.

\section{DISCUSSION}

The present experiment provides information regarding two independent but related learning phenomena, both associated with the performance of animals in $\mathrm{Fl}$ schedules. First, operant performance early in the FI cycle is known to increase when the prior cycle ends in surprising nonreward. This reinforcement-omission effect has been attributed to various factors, including disinhibition of performance by the removal of the reinforcer, invigoration of performance by primary frustration, and the incomplete reset of an internal clock critical for estimating the length of the interval (see the introduction for references). Another potential explanation tested in the present experiment was based on the possibility that the presentation of a reinforcer induces a transient state of satiety that depresses performance. This demotivation account has not been explicitly tested in the context of the reinforcement-omission effect (see Zimmerman, 1971, for results consistent with a demotivation account), but it has been a prominent hypothesis in studies of the frustration effect, a related phenomenon which does not involve timing but which shares with the present procedure the unexpected omission of reinforcers (e.g., Dudley \& Papini, 1995, 1997). These accounts were tested in Experiment 1 by manipulating the interval between successive cycles. Whereas the disinhibition and timing hypotheses predict no effect of changes in the ITI, both primary frustration and demotivation predict that an increase in the ITI should diminish or abolish the reinforcement-omission effect. This is so because both primary frustration and demotivation are assumed to be transient states that spontaneously decay in time. The results supported the demotivation account over any of the others; the critical evidence came from the fact that the reinforcement-omission effect was abolished by an increase in the ITI because the performance of animals after $\mathrm{R}$ cycles increased. The most parsimonious explanation is, therefore, that at least in some cases (see Kello, 1972, for an exception), the reinforcement-omission effect results from a depression of operant performance immediately after receiving a reinforcer caused by a transient state of demotivation. A more complete test of the primary frustration hypothesis demands an assessment of performance following $\mathrm{N}$ cycles in which the nonreinforcement episode is either unexpected, and hence presumably frustrating, or expected, and hence not frustrating (see, e.g., Scull, Davies, \& Amsel, 1970).

Second, operant performance in unusually long cycles typically peaks near the time at which the reinforcer is delivered in regular cycles. The results of Experiment 2 suggest that peak performance does not reflect exclusively the ability of animals to estimate the duration of an interval, as it is usually implied in the use of the peak procedure (see, e.g. $\mathrm{S}$. Roberts, 1981). Obviously, timing is involved in peak performance, since, when peak performance emerged in Phase 4, the response distribution tended to peak at a value close to that corresponding to the time when the reinforcer is delivered in training cycles. Nonetheless, these data suggest that the animal's ability to estimate the duration of an interval emerges behaviorally only with the engagement of some additional process that allows for performance to decrease during the second half of the test cycle. Simple temporal generalization decrement cannot explain peak performance, as is clearly shown in the test cycle performance during Phases $1-3$. In these phases, pigeons were suddenly shifted to an unusually long FI interval in the test cycles and yet showed no evidence of performance decrement after the 16 th second, when reinforcement had always been scheduled in training cycles.

One obvious candidate for a mechanism capable of producing the performance decrement late in the FI cycle would be anticipatory frustration. Initially, animals tend to respond during the unusually long intervals of test cycles until eventually the cycle ends and primary frustration occurs because of unexpected nonreward. As training continues, and given a sufficient number of $\mathrm{N}$ test cycles, the animal could learn to anticipate the frustrating outcome of cycles extending beyond the training $\mathrm{FI}$ interval. That anticipatory process would have a suppressive effect on ongoing operant performance (Amsel, 1992). The problem with this hypothesis is the lack of support for the presence of primary frustration to account for the reinforcement-omission effect in Experiment 1 . There is no basis to argue that primary frustration is present under the training conditions used in the present experiment, although there is some indication in the published literature that pigeons, as well as other species, find unexpected nonreward aversive (see Papini \& Dudley, 1997). Moreover, in a series of recent experiments using widely spaced training conditions (one trial per day), pigeons failed to show any indication that operant keypecking performance can be controlled by anticipatory frustration (Papini, 1997). These experiments involved unexpected shifts in reinforcement conditions, including shifts from large or small magnitudes to extinction and shifts from a large to a small magnitude. The pigeon's operant performance after the shift was directly related to the magnitude of reinforcement before the shift and not inversely related as predicted by frustration theory (Amsel, 1992). This is not an uncommon outcome in analogous experiments with lower vertebrates, but it is a different pattern of results from that typically obtained with mammalian species (Papini, Muzio, \& Segura, 1995).

It is possibie, therefore, that peak performance under the present conditions involves mechanisms other than the ones postulated by frustration theory. Animals not only can learn to estimate a particular interval, but also are able to discriminate the target interval from longer (or shorter) intervals (see, e.g., Santi, Weise, \& Kuiper, 1995). Given the extensive exposure to training and test cycles typical of timing experiments, it is reasonable to think of these two FI values as the $\mathrm{S}+$ and $\mathrm{S}-$ of a regular discrimination task (i.e., a time-based discrimination). In an autoshaping experiment with pigeons, Fernández-Serra, Arias-Holgado, and Gómez-Sancho (in press) found that peak performance gradually developed in the course of training, and they suggested that peak performance involved a discrimination between a 32-sec-long interval, the training cycle, and a 64-sec-long interval, the test cycle (see also Kirkpatrick-Steger, Miller, Betti, \& Wasserman, 1996). This explanation requires that time-related stimuli (e.g., sensory and/or mnemonic aftereffects of the stimuli associated with the start of the interval) acquire excitatory strength that initially generalizes and subsequently decreases with sufficient $\mathrm{S}-$ training. Another possibility is that $\mathrm{S}$ - stimuli acquire inhibitory strength, thus leading to response suppression late in the FI cycle. Peak performance could arise in Phase 4 as a result of differential excitation of discriminable time-related stimuli, or as the result of an interplay of excitatory and inhibitory gradients (see, e.g., Spence, 1936). When original training conditions were restored, as in Phase 5, either generalized excitation from $S+$ to $S-$ or extinction of inhibition in $S-$ could account for the re-emergence of the pattern of performance observed in Phases 1-3. It should be noted that reduced generalized excitation and inhibitory conditioning are usually difficult to distinguish as mechanisms of response suppression (Papini \& Bitterman, 1993).

One corollary of the present results is that because FI performance involves a multiplicity of processes, caution should be exercised in interpreting the effect of independent variables on peak performance purely in terms of a timing theory. Drug and lesion manipulations that are thought 
to affect some aspect of the timing process (see, e.g., Church, Broadbent, \& Gibbon, 1992) may produce their effects by influencing associative, motivational, and/or emotional processes on which FI performance also seems to depend.

\section{REFERENCES}

AMSEL, A. (1992). Frustration theory. Cambridge: Cambridge University Press.

Church, R. M., Broadbent, H. A., \& Gibbon, J. (1992). Biological and psychological description of an internal clock. In I. Gormezano \& E. A. Wasserman (Eds.), Learning and memory: The behavioral and biological substrates (pp. 105-128). Hillsdale, NJ: Erlbaum.

Dudley, R. T., \& PAPINI, M. R. (1995). Pavlovian performance of rats following unexpected reward omissions. Learning \& Motivation, 26, 63-82.

Dudley, R. T., \& PAPINI, M. R. (1997). Amsel's frustration effect: A Pavlovian replication with control for frequency and distribution of rewards. Physiology \& Behavior, 61, 627-629.

Fernández-Serra, F., Arias-Holgado, M. F., \& Gómez-Sancho, L. E. (in press). Discriminación y generalización temporal en automoldeamiento: Transición y estado estable. Acta Comportamentalia.

KeLLO, J. E. (1972). The reinforcement-omission effect on fixed-interval schedules: Frustration or inhibition? Learning \& Motivation, 3, 138147.

Kirkpatrick-Steger, K., Miller, S. S., Betti, C. A., \& Wasserman, E. A. (1996). Cyclic responding by pigeons on the peak timing procedure. Journal of Experimental Psychology: Animal Behavior Processes, 22, 447-460.

LeJeUne, H., \& WEARden, J. H. (1991). The comparative psychology of fixed-interval responding: Some quantitative analyses. Learning \& Motivation, 22, 84-111.

Mellon, R. C., Leak, T. M., Fairhurst, S., \& Gibbon, J. (1995). Timing processes in the reinforcement-omission effect. Animal Learning \& Behavior, 23, 286-296.

PAPINI, M. R. (1997). Role of reinforcement in spaced-trial operant learn- ing in pigeons (Columba livia). Journal of Comparative Psychology, $111,275-285$.

PAPINI, M. R., \& Bitterman, M. E. (1993). The two-test strategy in the study of inhibitory conditioning. Journal of Experimental Psychology: Animal Behavior Processes, 19, 342-352.

PaPINI, M. R., \& DudLEY, R. T. (1997). Consequences of surprising reward omissions. Review of General Psychology, 1, 175-197.

Papini, M. R., Muzio, R. N., \& Segura, E. T. (1995). Instrumental learning in toads (Bufo arenarum): Reinforcer magnitude and the medial pallium. Brain, Behavior \& Evolution, 46, 61-71.

ROBERTS, S. (1981). Isolation of an internal clock. Journal of Experimental Psychology: Animal Behavior Processes, 7, 242-268.

Roberts, W. A., Cheng, K., \& CoHEN, J. S. (1989). Timing light and tone signals in pigeons. Journal of Experimental Psychology: Animal Behavior Processes, 15, 23-35.

SANTI, A., Weise, L., \& Kuiper, D. (1995). Memory for event duration in rats. Learning \& Motivation, 26, 83-100.

Scull, J., Davies, K., \& Amsel, A. (1970). Behavioral contrast and frustration effect in multiple and mixed fixed-interval schedules in the rat. Journal of Comparative Psychology, 71, 478-483.

SPENCE, K. W. (1936). The nature of discrimination learning in animals. Psychological Review, 43, 427-449.

STADDON, J. E. R. (1970). Temporal effects of reinforcement: A negative "frustration" effect. Learning \& Motivation, 1, 227-247.

Staddon, J. E. R., \& INNis, N. K. (1966). An effect analogous to "frustration" on interval reinforcement schedules. Psychonomic Science, 4, 287-288.

STAdDON, J. E. R., \& INNIS, N. K. (1969). Reinforcement omission on fixed-interval schedules. Journal of the Experimental Analysis of Behavior, 12, 689-700.

Zimmerman, D. W. (1971). Rate changes after unscheduled omission and presentation of reinforcement. Journal of the Experimental Analysis of Behavior, 15, 261-270.

(Manuscript received October 2, 1996; revision accepted for publication June 9, 1997.) 